\title{
Evidence of enhanced formation episodes in the Galactic open cluster system
}

\author{
A. E. Piatti \\ Instituto de Astronomía y Física del Espacio, CC 67, Suc. 28, 1428 Buenos Aires, Argentina \\ e-mail: andres@iafe.uba.ar \\ Received 8 February 2010 / Accepted 6 April 2010 \\ ABSTRACT

\begin{abstract}
Aims. The exciting debate about the existence of signs of enhanced formation of Galactic open clusters (OCs) is revisited here on the basis of a revised age distribution.

Methods. The data were taken from the recently updated 2009 version of the Dias et al.'s 1787 OC catalogue.

Results. We found that the present OC's age distribution presents two primary excesses at $t \sim 10-15$ Myr and 1.5 Gyr, which are signs of enhanced formation episodes similar to those that occurred in other galaxies (e.g., M 51, NGC 1705). When restricting the OC sample to those located in the solar neighbourhood, with the aim of avoiding incompleteness effects, we also find that these clusters are engraved with clear signs of enhanced formation at both ages.
\end{abstract}

Key words. galaxies: star clusters: general - open clusters and associations: general

\section{Introduction}

It is known that signs of star cluster formation from bursting episodes have been found in some galaxies. For instance, the enlarged Small Magellanic Cloud (SMC) star cluster sample of Piatti et al. (2007a) appears to strengthen the observed agemetallicity relation (AMR), making it more consistent with the bursting star formation history of Pagel \& Tautvaišienè (1998) than with a simple closed system with continuous star formation under the assumption of chemical homogeneity (Da Costa \& Hatzidimitriou 1998). Previous studies favouring the bursting star formation scenario in the SMC are those of Mighell et al. (1998), Rich et al. (2000), Bekki et al. (2004), and Piatti et al. (2005), among others.

On the other hand, Piatti et al. (2003) also use an homogenous Large Magellanic Cloud (LMC) star cluster sample to show that the bursting model by Pagel \& Tautvaišiene (1998) for the LMC fits the star cluster AMR better than the closed-box enrichment model computed by Geha et al. (1998) using the star formation history of Holtzman et al. (1997). These results show star cluster excesses within a relatively narrow age range at $t \sim 2 \mathrm{Gyr}$ (see also Piatti et al. 2007b, 2009). In addition, evidence of gravitational triggered episodes of cluster formation due to the most recent close interaction between both Magellanic Clouds (MCs) was found in the cluster age distributions (Chiosi et al. 2006).

In M 33 - one of the three spiral galaxies in the Local Group - some authors have found a couple of peaks in their cluster age histograms (e.g. Chadar et al. 2001; Ma et al. 2004, and references therein) corresponding to different enhanced cluster formation epochs. For star clusters in M 51, Hwang \& Lee (2009) conclude that their formation rate increased significantly during the period of 100-250 Myr ago, which roughly coincides with the epoch of dynamical encounters of two galaxies, NGC 5194 and NGC 5195, expected by theoretical models. The age distribution of the star clusters in M51 also shows two peaks about 100 Myr and 250 Myr ago. Annibali et al. (2009) also confirm two strong bursts of star formation in the late-type dwarf galaxy NGC 1705. They obtained $H S T$ photometry and, with the method of the synthetic colour-magnitude diagrams, detected an older burst between 10 and 15 Myr ago, coeval to the age of the central super star clusters and a younger burst started 3 Myr ago, which is still active. Finally, Yin et al. (2009) find that star clusters in M31 have undergone a more active formation history, even in the recent past, as suggested by observations of a "head-on" collision with the neighbouring M 32 galaxy about 200 Myr ago.

As far as the Galaxy evolution is concerned, the possible tidal interaction effects in the Milky Way (MW) disk have not been considered for long. Indeed, many models have been developed to explain the chemical evolution of the MW, but have only invoked gas infall for the formation of the Galactic disk (Chiappini et al. 1997). Sometimes, the star formation efficiency was assumed to be constant along the disk (Colavitti et al. 2008), whereas other studies use a model in which the infall timescale of the disk varies with radius (Boissier \& Prantzos 1999). Although more recently some other theoretical approaches have considered the interaction between the MCs and the MW (Tsuchiya 2002; Bekki \& Chiba 2007; Shattow \& Loeb 2009), they mainly focus on the kinematical arena. For instance, Tsuchiya (2002) examined the contribution of the LMC to the Galactic warp by using $N$-body simulations, and Shattow \& Loeb (2009) find that the past orbit of the LMC is naturally confined within the virial boundary of the MW.

Then open clusters entered the picture. When the Galactic disk was formed, OCs have certainly been affected by the nucleosynthesis processes (Robin et al. 2004), the radial chemical composition gradient (Chen et al. 2003), the lack of a clear AMR (Friel et al. 2002), the appearance of a tidal warp (Vázquez et al. 2008), etc. Thus, bearing in mind the evidence of bursting cluster formation in other galaxies, we would also expect that 
OCs show signs of enhanced formation if such an event occurred in the MW. Several and varied observational and theoretical consequences for the formation and evolution of the Galactic disk have been suggested. Salaris et al. (2004) find that the cluster formation and destruction processes are not, apparently, in direct correlation with the Galactocentric distance, and Frinchaboy et al. (2004) argue that the tidal accretion of a dwarf satellite galaxy on a low inclination orbit appears to be a plausible explanation for the origin of the outer, old OCs. Chen et al. (2001) also conclude in favour of a scenario in which the thick disk was formed through the heating of a preexisting thin disk, with the heating mechanism being the merging of a satellite galaxy.

However, to date, the most frequently picture of the age distribution of Galactic OCs results in a combination of two exponential-decay curves representing the young and old OC populations (Janes \& Phelps 1994; Bonatto et al. 2006). It does not account for any excess of OCs that could have formed during bursting formation episodes. This Letter tries to show that the Galactic OC system is also marked by enhanced formation events, which are frequently associated with galaxy interactions. To arrive at this result, we performed a sound analysis of the OC's age distribution, showing that past and recent OC enhancements exist. Since previous cluster age histograms had been drawn by adopting arbitrary age intervals, without any justification for the chosen bin size, they had not seen the jumps/peaks of the bursts (or enhanced formation). Here, we explore the consequences of adopting different bin sizes and propose using a more robust criterion to define the age intervals, hence to obtain a genuine $\mathrm{OC}$ age distribution. Section 2 deals with a revisited OC's age distribution and presents evidence of enhanced OC formation that could be interpreted as bursting episodes. In Sect. 3 we provide additional clues to this enhanced OC formation from the comparison with the cluster age distributions of other galaxies. Finally, Sect. 4 summarizes our findings and conclusions.

\section{Age distribution of Galactic open clusters}

Janes \& Phelps (1994), Bonatto et al. (2006, hereafter BKBS), and $\mathrm{Wu}$ et al. (2009, hereafter WZMD), among others, have examined the OC's age distribution. As mentioned above, they have found OCs with ages spanning the range $1 \mathrm{Myr}-9 \mathrm{Gyr}$ with two typical timescales that resemble the timescales of the thin and thick Galactic disks. BKBS found to this result from a thorough study of the properties of the Galactic disk based on 654 OCs distributed within a region of $\sim 5 \mathrm{kpc}$ from the Sun, using the WEBDA $^{1}$ database. On the other hand, WZMD employed a recently updated version (February 2009) of the Dias et al. OC catalogue (2002). This catalogue provides information on the fundamental parameters for 1787 objects and includes the previous catalogues of Lyngå (1987) and Mermilliod (1995, in the WEBDA database). New objects and data that were not present in the previous catalogues have now been included in this one. However, from an astrophysical point of view, the number of OCs with age estimates are nearly the same in both studies.

In general, the histograms of the OC's age distribution have been built using a fixed age interval. BKBS used a bin size of $200 \mathrm{Myr}$ over the whole age range $(\Delta t \sim 9 \mathrm{Gyr})$. However, with an age interval of $200 \mathrm{Myr}$, it is impossible to distinguish the intrinsic fluctuations in the OC's age distribution during the past $100 \mathrm{Myr}$, which are seen in bin sizes of $\sim 10 \mathrm{Myr}$. On the other hand, employing bin sizes of $200 \mathrm{Myr}$ results in a nearly subsampled OC's age distribution for ages over $\sim 4$ Gyr. The large

1 http://obswww . unige. ch/webda difference among the ages of OCs led WZMD varied the age intervals and used bin sizes of $100 \mathrm{Myr}$ for OCs younger than 1 Gyr and 500 Myr for OCs older than 1 Gyr (see their Fig. 2).

We also produced OC's age histograms using the updated version $^{2}$ of the Dias et al. OC catalogue (2002) and age intervals of $50 \mathrm{Myr}, 100 \mathrm{Myr}, 200 \mathrm{Myr}$, and $500 \mathrm{Myr}$, respectively. In each histogram we found peculiar features not seen in the remaining ones. These examples show that a fixed age bin size is not appropriate for yielding the intrinsic age distribution, since the result depends on the chosen age interval. A more robust age interval should be the one whose width is a measure of the age errors of the clusters in that interval. This would lead chosing very narrow bins for young clusters and relatively broader age bins for the older ones.

With the aim of building an age histogram that tightly reproduces the intrinsic OC's age distribution, we take the uncertainties in the age estimates into account to define the age intervals in the whole OC age range. Thus, we produce a more appropriate sampling of the OCs per age interval than is built using a fixed bin size, since we include in each bin a number of clusters whose age errors are close to the size of this bin. Indeed, the age errors for very young OCs are a couple of Myr, while those for the oldest OCs are at least of a few Gyr. Therefore, smaller bins are apropriate for young clusters, whereas larger bins are suitable for the old clusters. We then searched the literature to find that typical age errors are $0.10 \lesssim \Delta \log (t) \lessgtr 0.20$. Therefore, we produced a revised age histogram by setting the bin sizes according to this logarithmic law, which traces the variation in the derived age uncertainties in terms of the OC ages. We used intervals of $\Delta \log (t)=0.20$, but a slightly noisy histogram would be obtained if we used $\Delta \log (t)=0.10$. The subdivision of the whole age range in age intervals of different sizes can be performed on a observational-based foundation, since they are a measure of the typical OC age errors for each age range. Figure 1 shows the resulting age histogram compared to those by BKBS and WZMD. We also included the errorbars produced by a Poisson statistics. As can be seen, we can unveil fiducial characteristics of the OC's formation history during the past tens of Myr, and appropriately group the older ones as well.

\section{Analysis of the $\mathrm{OC}$ age distribution}

Figure 1 depicts a close-up view at the younger ages along with an integral picture of the older ones, thus spanning the whole age range in detail. The close-up panel reveals an important excess of OCs at $\sim 10-15 \mathrm{Myr}$, which curiously arises after a period (30 Myr $\lesssim t \lesssim 100 \mathrm{Myr}$ ) of decreasing OC formation, overtaking the previously simple picture that $2 / 3$ of the known OCs were born during the last $100 \mathrm{Myr}$ (WZMD). The figure also presents very interesting features. Firstly, there is an abrupt jump at $\sim 1.5 \mathrm{Gyr}$, which largely doubles the number of older OCs counted in the adjacent bin, and continues with a relatively steady OC formation rate for the adjacent younger bins (300 Myr $\lesssim t \lesssim 1500 \mathrm{Myr}$ ). The relatively wide age interval at the jump bin $(t \sim 1.0-1.5 \mathrm{Gyr})$ reflects the size of the age errors at that age, rather than a measure of the duration of the burst or triggered OC formation process. Secondly, this noticeably increased level in the number of OCs seems to keep up on average until the last hundred Myr, when a change in the slope of the histogram takes place $(t \sim 100 \mathrm{Myr})$. Both primary excesses $(t \sim 10-15 \mathrm{Myr}$ and $1.5 \mathrm{Gyr})$ are signs of enhanced formation

\footnotetext{
${ }^{2}$ February 2009; http://www . astro.iag.usp.br/ wilton
} 
A. E. Piatti: Open cluster enhanced formation episodes

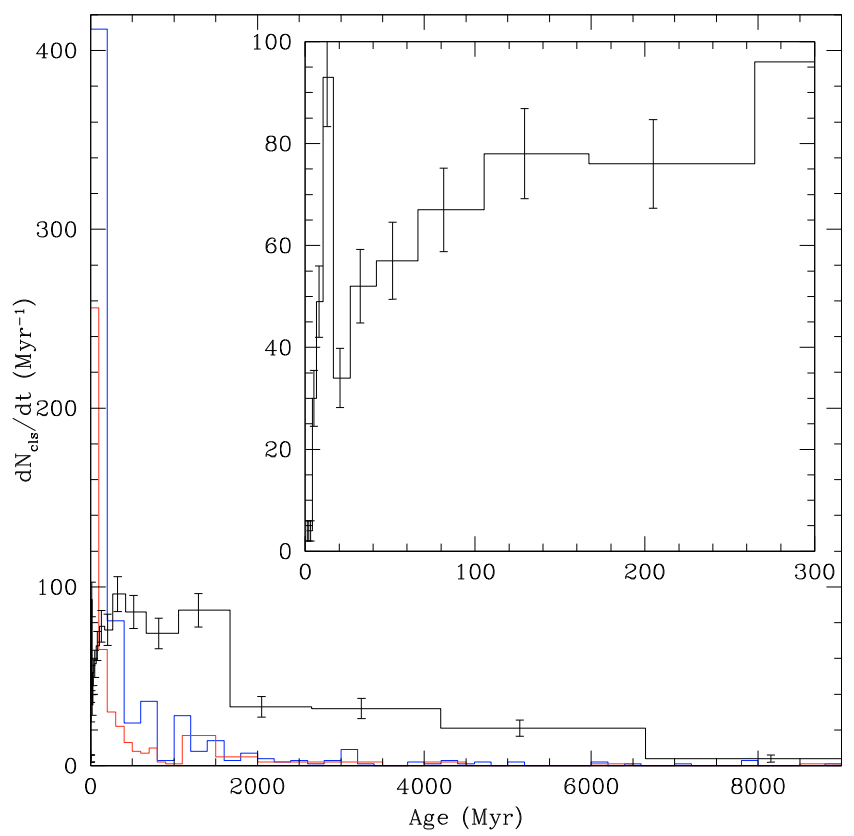

Fig. 1. OC's age histograms obtained by BKBS (2006), WZMD (2009) and by us represented with blue, red, and black solid lines, respectively.

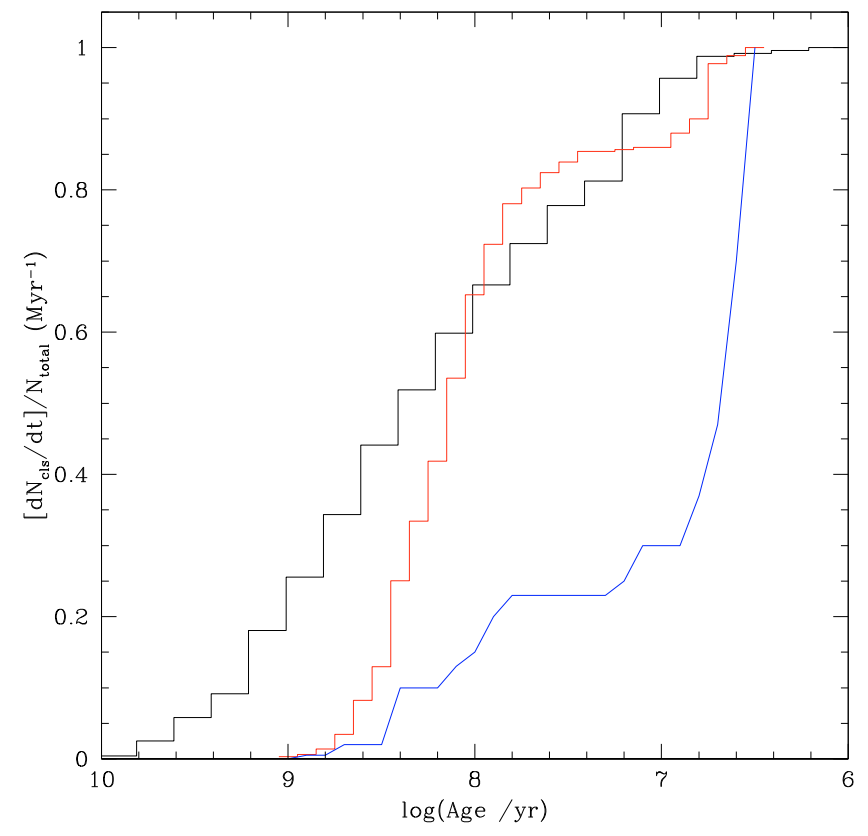

Fig. 2. Normalized cumulative age distribution for Galactic OCs (black line), and star clusters in M 51 (red) and NGC 4395, blue solid line. episodes, similar to the bursting cluster formation events occurring in other galaxies (see Sect. 1).

With the aim of confirming a bursting-like OC formation process in the MW in contrast to a closed-box scenario where the OC formation is at a constant rate, we compared the cumulative age distribution of the Galactic OCs with those of star clusters in the unperturbed spiral galaxy NGC 4395 (Mora et al. 2009) and in M 51, which shows vestiges of star cluster bursts (Hwang \& Lee 2009). The result is depicted in Fig. 2, wherein we normalized the age distributions for comparison purposes. The comparison is strictly valid for clusters younger than $\sim 1 \mathrm{Gyr}$, which is the range observed by Mora et al. (2009) and Hwang \& Lee (2009). As can be seen, the cumulative OC age distribution is more like that of M 51 than NGC 4395. Therefore, we conclude that the OC formation rate in the Milky Way has been enhanced during its lifetime.

We recall that much more work is needed to confirm whether the enhanced OC formation would have taken place across the entire MW disk, more concentrated in the Galactic centre, more concentrated in the outer parts of the MW disk, or even azimuthaly uniform. Other questions still remain, as whether the enhanced OC formation were triggered by interaction with the MCs (McClure-Griffiths et al. 2008; Shattow \& Loeb 2009), accretion of a dwarf satellite galaxy (Frinchaboy et al. 2004), infall of high-velocity gas clouds (Lockman et al. 2009), etc.

\subsection{Completeness of the age distribution}

To confirm the physical nature of the OC's excesses at 10-15 Myr and 1.5 Gyr, we considered the possibility that the Dias et al. OC catalogue (2002) could be biased by incompleteness. Wielen (1971) studied the age distribution of Galactic OCs from the catalogues of Becker \& Fenkart (1971) and Lindoff (1968). His samples contain 70 and 59 OCs within a Galactic distance of $1 \mathrm{kpc}$ from the Sun. Wielen showed that his list of clusters is not complete, but that it provides representative sampling of the OC's age distribution. Later, Boutloukos \& Lamers (2003) used the Wielen's OC compilation to estimate the OC disruption time, including the evolutionary fading and the mass-dependent dynamical cluster dissolution by tidal effects, as well as the mass-independent dissolution of star clusters in their very early stages (infant mortality). They conclude that this OC sample is not biased towards the younger ages.

More recently, BKBS have performed simulations of the OC completeness in the WEBDA database. They conclude that this sample can be taken as statistically representative of the OCs of Trumpler types I to III. Furthermore, the resulting completeness-corrected age distribution and fitted timescales retain the basic features of the observed one within the uncertainties. They confirmed these results particularly for their restricted OC sample with heliocentric distances below $1 \mathrm{kpc}$. Finally, to estimate the completeness of the OCs in the updated version of the Dias et al. catalogue (the OC sample used in this Letter), WZMD analysed the volume density of OCs as a function of the heliocentric distance to find that their OC sample is a representative subsample of the currently observed OCs in the Galaxy.

The previous results allow us to conclude that our findings should not be affected by incompleteness and that a restricted subsample of OCs located within $1 \mathrm{kpc}$ from the Sun (solar neighbourhood) should guarantee completeness. For that reason, even though the age distribution of these OCs could not account for the magnitude of the effects produced by the triggered enhanced OC formation in the MW disk, it may still be valuable to compare it with those derived here for completeness purposes. Figure 3 shows the resulting age histograms for the whole OC sample (top), the OC located in the solar neighbourhood (middle), and the star clusters in M 51 (bottom). The cumulative histogram of M 51 (Fig. 2) was obtained from this graph. As can be seen, both excesses of OCs ( $t \sim 10-15 \mathrm{Myr}$ and 1.5 Gyr) are clearly visible, which means that the solar neighbourhood was also reached by the enhanced formation episodes. 


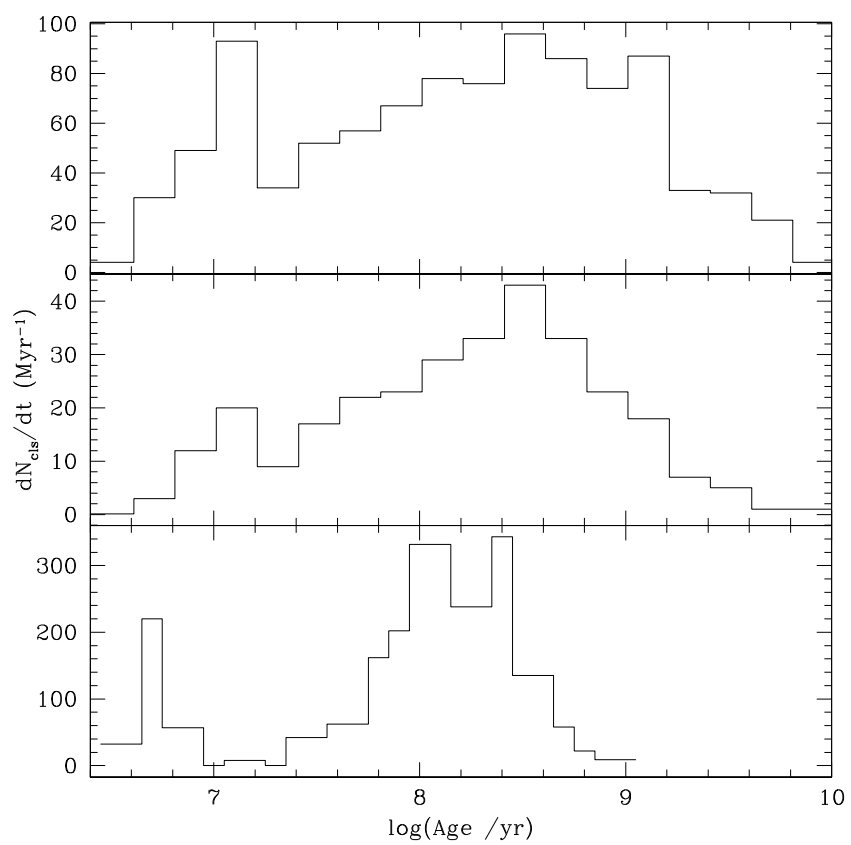

Fig. 3. Age histograms for the whole OC sample (top), OC located in the solar neighbourhood (middle), and star clusters in M 51 (bottom).

\section{Conclusions}

Based on the updated version of the Dias et al. (2002) OC catalogue, we analysed the OC's age distribution for signs of enhanced formation rates possibly triggered by bursting mechanims. The results of this analysis led us to the following main conclusions.

i) By adopting an age interval varying in terms of the age according to a logarithmic law, we built a revised OC's age histogram, which proved to trace the intrinsic OC's age distribution more properly. The revised age distribution can be considered to statistically represent the OC population.

ii) The present OC's age distribution presents two primary excesses at $t \sim 10-15 \mathrm{Myr}$ and $1.5 \mathrm{Gyr}$, which are possible signs of enhanced formation episodes, similar to those in other galaxies (e.g., M 51, NGC 1705).

iii) When restricting the OC sample to those located in the solar neighbourhood with the aim of avoiding incompleteness effects, we also find that these clusters show clear signs of enhanced formation at both ages.

Acknowledgements. This work was partially supported by the Argentinian institution CONICET. We thank the reviewer whose comments helped us to improve the manuscript.

\section{References}

Annibali, F., Tosi, M., Monelli, M., et al. 2009, AJ, 138, 169

Becket, W., \& Fenkart, R. 1971, A\&AS, 4, 241

Bekki, K. \& Chiba, M. 2007, MNRAS, 381, L16

Bekki, K., Couch, W. J., Beasley, M. A., et al. 2004, ApJ, 610, L93

Boissier, S., \& Prantzos, N. 1999, MNRAS, 307, 857

Bonatto, C., Kerber, L. O., Bica, E., \& Santiago, B. X. 2006, A\&A, 446, 121 (BKBS)

Boutloukos, S. G., \& Lamers, H. J. G. L. M. 2003, MNRAS, 338, 717

Chandar, R., Bianchi, L., \& Ford, H. C. 2001, A\&A, 366, 498

Chen, B., Stoughton, C., Smith, J. A., et al. 2001, ApJ, 553, 184

Chen, L., Hou, J. L., \& Wang, J. J. 2003, AJ, 125, 1397

Chiappini, C., Matteucci, F., \& Gratton, R. 1997, ApJ, 477, 765

Chiosi, E., Vallenari, A., Held, E. V., Rizzi, L., \& Moretti, A. 2006, A\&A, 452, 179

Colavitti, E., Matteucci, F., \& Murante, G. 2008, A\&A, 483, 401

Da Costa, G. S., \& Hatzidimitriou, D. 1998, AJ, 115, 1934

Dias, W., Alessi, B. S., Moitinho, A., \& Lepine, J. R. D. 2002, A\&AS, 141, 371

Friel, E. D., Janes, K. A., Tavarez, M., et al. 2002, AJ, 124, 2693

Frinchaboy, P. M., Majewski, S. R., Crane, J. D., et al. 2004, ApJ, 602, L21

Geha, M. C., Holtzman, J. A., Mould, J. R., et al. 1998, AJ, 115, 1045

Holtzman, J. A., Mould, J. R., Gallagher, J. S., III, et al. 1997, AJ, 113, 656

Hwang, N., \& Lee, M. G. 2009, ApJ, 709, 411

Janes, K., \& Phelps, R. L. 1994, AJ, 108, 1773

Lindoff, U. 1968, Ark. Astron., 5, 1

Lockman, F. J., Benjamin, R. A., Heroux, A. J., \& Langston, G. I. 2008, ApJ, 679, L21

Lyngå, G. 1987, Catalogue of Open Cluster Data, Strasbourg: Centre de Données Stellaires

Ma, J., Zhou, X., \& Chen, J. 2004, A\&A, 413, 563

McClure-Griffiths, N. M., Staveley-Smith, L., Lockman, F. J., et al. 2008, ApJ, 673, L143

Mermilliod, J.-C. 1995, in Information and On-Line Data in Astronomy, ed. D. Egret, \& M. A. Albrecht (Dordrecht: Kluwer), 127

Mighell, K. J., Sarajedini, A., \& French, R. S. 1998, AJ, 116, 2395

Mora, M. D., Larsen, S. S., Kissler-Patig, M., Brodie, J. P., \& Richtler, T. 2009, A\&A, 501, 949

Pagel, B. E. J., \& Tautvaišienè, G. 1998, MNRAS, 299, 535

Piatti, A. E., Bica, E., Geisler, D., \& Clariá, J. J. 2003, MNRAS, 344, 965

Piatti, A. E., Sarajedini, A., Geisler, D., Seguel, J., \& Clark, D. 2005, MNRAS, 358, 1215

Piatti, A. E., Sarajedini, A., Geisler, D., Gallart, C., \& Wischnjewsky, M. 2007a, MNRAS, 381, L84

Piatti, A. E., Sarajedini, A., Geisler, D., Gallart, C., \& Wischnjewsky, M. 2007b, MNRAS, 382, 1203

Piatti, A. E., Geisler, D., Sarajedini, A., \& Gallart, C. 2009, A\&A, 501, 585

Rich, R. M., Shara, M., Fall, S. M., \& Zurek, D. 2000, AJ, 119, 197

Robin, A. C., Reylé, C., Derrière, S., \& Picaud, S. 2004, A\&A, 416, 157

Salaris, M., Weiss, A., \& Percival, S. M. 2004, A\&A, 414, 163

Shattow, G., \& Loeb, A. 2009, MNRAS, 392, L21

Tsuchiya, T. 2002, New Astron., 7, 293

Vázquez, R. A., May, J., Carraro, G., et al. 2008, ApJ, 672, 930

Wielen, R. 1971, A\&A, 13, 309

Wu, Z.-Y., Zhou, X., Ma, J., \& Du, C.-H. 2009, MNRAS, 399, 214 (WZMD)

Yin, J., Hou, J. L., Prantzos, N., et al. 2009, A\&A, 505, 497 